\title{
Invaginación intestinal por doble GIST
}

\author{
Intestinal intussusception due to double GIST
}

Eduardo A. Rodríguez-Martínez ${ }^{1}$, Silvia Carbonell-Morote ${ }^{1}$, Emilio Ruiz-de la Cuesta ${ }^{1}$, Ester Gracia-Alegría ${ }^{1}$, Celia Villodre-Tudela ${ }^{1}$ y Félix Lluis-Casajuana ${ }^{1}$

Mujer de 67 años, con antecedentes de melanoma nodular en miembro inferior derecho, artritis reumatoide en tratamiento con infliximab-metotrexate, anticoagulante lúpico $+\mathrm{y}$ osteoporosis. Ingresa por cuadro de melena y astenia de 2 semanas de evolución. La analítica mostró HB 7,7 g/dL; Hcto 25,3\%; 13.000 leucocitos con $11,00 \times 10^{3} / \mu \mathrm{L}$ neutrófilos. $\mathrm{La}$ coagulación APTT alargado en contexto de coagulante lúpico conocido.

Tras colonoscopia normal y gastroscopia normal se realizó TC toracoabdominopélvico que evidenció hallazgos compatibles con invaginación intestinal por posible neoplasia en asa proximal de yeyuno sin poder descartar metástasis de melanoma (Figuras 1 y 2). Se decidió intervención quirúrgica hallándose asa de yeyuno proximal dilatada con invaginación dependiente de tumoración intraluminal. Se realizó resección en bloque de unos $30 \mathrm{~cm}$ de yeyuno

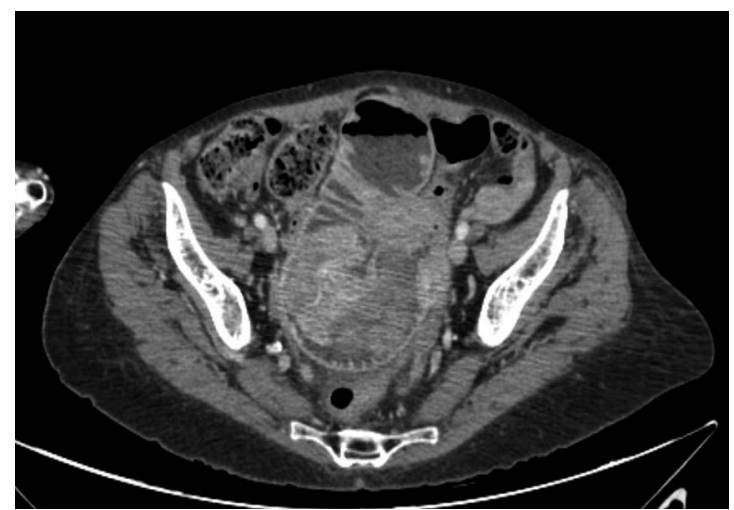

Figura 1. TC de pelvis corte axial, con hallazgo de invaginación intestinal. incluido el mesenterio y los ganglios linfáticos regionales (Figura 3).

El postoperatorio transcurrió sin incidencias siendo alta al sexto día de la intervención.

El informe anatomopatológico definitivo informó de dos lesiones de $1,5 \mathrm{~cm}$ y $11,6 \mathrm{~cm}$ de diámetro, tipo histológico mixto (fusocelular-epitelioide). $\mathrm{Al}$ menos 7 mitosis $/ 5 \mathrm{~mm}^{2}$, G2 con un riesgo de metástasis de $90 \%$ (Figura 4).

La inmunohistoquímica confirmó la estirpe GIST (CKIT: positivo, S100: positivo, CD34: positivo) siendo negativos los marcadores de melanoma.

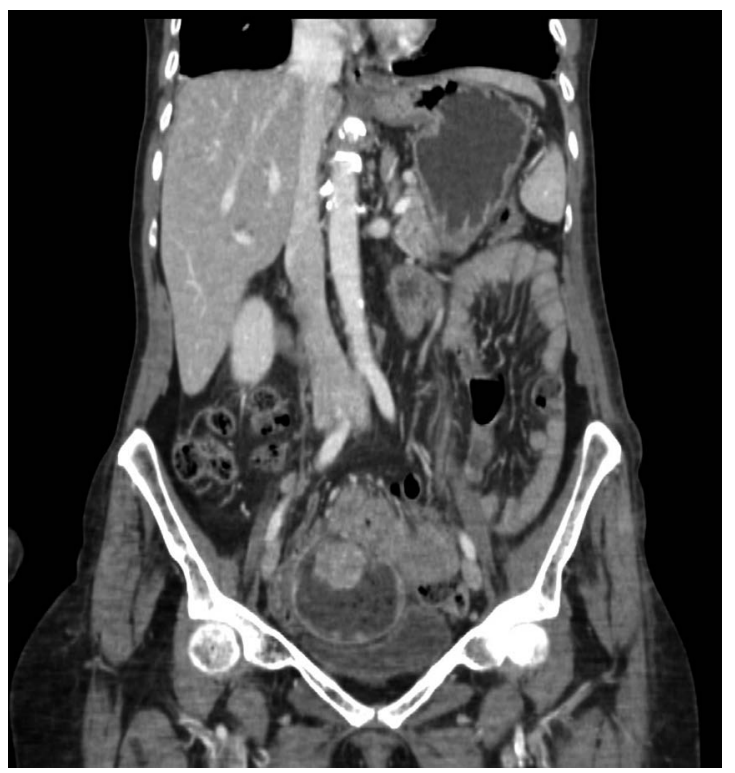

Figura 2. TC de abdomen y pelvis corte coronal con evidencia en pelvis, de intestino invaginado.
${ }^{1}$ Hospital General Universitario de Alicante. Alicante, España.

Recibido el 30 de octubre de 2019 y aceptado para publicación el 4 de noviembre de 2019 .

Correspondencia a: Dr. Eduardo A. RodríguezMartínez

edalroma@hotmail.com 


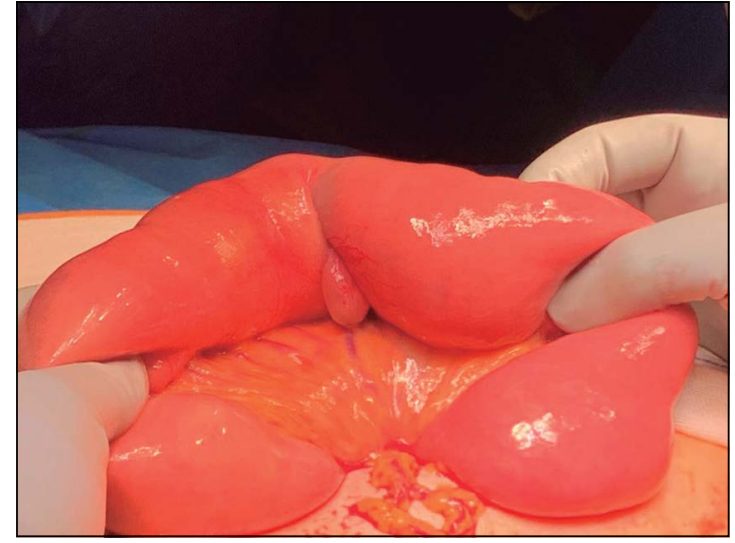

Figura 3. Pieza resecada.

\section{Responsabilidades éticas}

Protección de personas y animales. Los autores declaran que para esta investigación no se han realizado experimentos en seres humanos ni en animales.

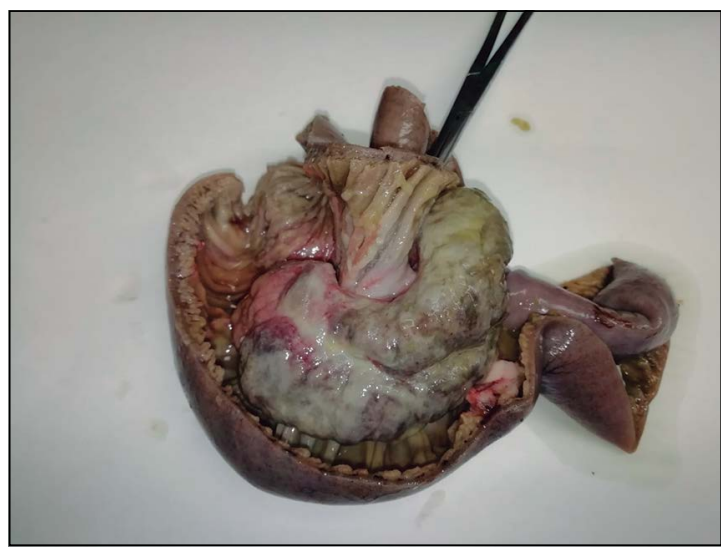

Figura 4. Pieza resecada abierta.

Confidencialidad de los datos. Los autores declaran que en este artículo no aparecen datos de pacientes.

Conflictos de interés: no hay.

\section{Bibliografía}

1. Fourati H, Hmaydia G, Harbi H, Mzali R, Mnif Z. Acute bowel obstruction revealing a pedunculated gastro-intestinal stroma tumor (GIST). PresseMed. 2016;45:792-4.

2. Massani M, Capovilla G, Ruffolo C, Bassi N. Gastrointestinal stromal tumour (GIST) presenting as a strangulated inguinal hernia with small bowel obstruction. BMJ Case Rep. 2017;2017:bcr2016217273. DOI:10.1136/bcr-2016-217273

3. Sorour MA, Kassem MI, GhazalAel-H, El-Riwini MT, Abu Nasr A.

Gastrointestinal stromaltumors
(GIST) relatedemergencies. Int J Surg. 2014;12:269-280. DOI:10.1016/j. ijsu.2014.02.004

4. Scola D, Bahoura L, Copelan A, Shirkhoda A, Sokhandon F. Getting the GIST: a pictorial review of the various patterns of presentation of gastrointestinal stromal tumors on imaging. AbdomRadiol (NY). 2017;42:1350-1364. DOI:10.1007/ s00261-016-1025-z.

5. Sadeghi P, Lanzon-Miller S. A jejunal GIST presenting with obscure gastrointestinal bleeding and small bowel obstruction secondary to intussusception. BMJ Case Rep. 2015;2015:bcr2014207650. DOI:10.1136/ bcr-2014-207650.

6. Rittenhouse DW, Lim PW, Shirley LA, Chojnacki KA. Gastroduodenal intussusception of a gastrointestinal stromal tumor (GIST): case report and review of the literature.

SurgLaparoscEndosc Percutan Tech. 2013;23:e70-3. DOI:10.1097/ SLE.0b013e31826d72d4

7. Gupta S, Punia RS, Kaushik R. Gastrointestinal stromal tumour of the colon presenting with intestinal obstruction. Indian J Cancer. 2004;41:1757. 